\title{
ADAPTIVE EXTERNAL TORQUE ESTIMATION BY MEANS OF TRACKING A LYAPUNOV FUNCTION
}

\author{
Hanspeter Schaub ${ }^{*}$, Rush D. Robinett ${ }^{\dagger}$ and John L. Junkins ${ }^{*}$ \\ A real-time method is presented to adaptively estimate three-dimensional \\ unmodeled external torques acting on a spacecraft. This is accomplished \\ by forcing the tracking error dynamics to follow the Lyapunov function \\ underlying the feedback control law. For the case where the external \\ torque is constant, the tracking error dynamics are shown to converge \\ asypmtotically. The methodology applies not only to the control law used \\ in this paper, but can also be applied to most Lyapunov derived feedback \\ control laws. \\ The adaptive external torque estimation is very robust in the presence of \\ measurement noise, since a numerical integration is used instead of a nu- \\ merical differentiation. Spacecraft modelling errors, such as in the inertia \\ matrix, are also compensated for by this method. Several examples illus- \\ trate the practical significance of these ideas
}

RECEIVED

MAR 151996

OSTI

\section{INTRODUCTION}

In most spacecraft control problems it is difficult to predict the external forces and torques acting on the body. These forces/torques could be due to aerodynamic drag in low Earth orbit, the solar wind, or any one of many other disturbances occurring in space. All these external disturbances are usually small in magnitude and are varying very slowly, but can be of consequence if high precision attitude tracking is required.

A method is presented to adaptively estimate the external torque in real-time, thus allowing the control law to compensate for it. This methods involves comparing the time histories of the theoretical and the measurement-based estimate of the Lyapunov function which underlies the control law design. Out of the difference of the Lyapunov functions, the external torque estimate is updated. Without this estimate, a feedback control of this type is not guaranteed to asymptotically track the desired motion if unmodeled external torques are present. The tracking errors will always converge to a finite offset associated with the magnitude of the external disturbance. How large this offset is depends on how large the feedback gains are. With the external torque compensation presented here, the feedback control law is able to asymptotically track a desired motion if estimation errors are negligible.

\footnotetext{
- Graduate Research Assistant, Aerospace Engineering Department, Texas A\&M University, College Station TX77843.

† Research Engineer, Sandia National Laboratories, Albuquerque, NM 87185.

₹ George Eppright Chair Professor of Aerospace Engineering, Aerospace Engineering Department, Texas A\&M University, College Station TX77843, Fellow AAS.
}

This work was supported by the United States Department of Energy

under Contract DE-AC04-94AL85000. 


\section{DISCLAMMER}

Portions of this document may be illegible in electronic image products. Images are produced from the best available original document. 
This adaptive external torque estimation method is presented using the globally stabilizing nonlinear Lyapunov tracking control law developed recently by Schaub, Robinett and Junkins ${ }^{1}$. The method itself is not tied to the specific Lyapunov function used in Ref. 1, analogous methods exist for any Lyapunov derived control law which guarantees asymptotic tracking in the absence of disturbance model errors. Some general stability and convergence analysis of the method are presented.

\section{PROBLEM FORMULATION}

\section{The Equations of Motion of a Rigid Spacecraft}

The rigid spacecraft dynamics with three reaction wheels of varying inertias $J_{i}$ is described below. Each wheel is aligned with one of the principal inertia axis. Let the inertia matrix $\mathfrak{I}$ contain the spacecraft and the transverse reaction wheel inertias and let the matrix $J$ be defined as

$$
J=\left[\begin{array}{ccc}
J_{1} & 0 & 0 \\
0 & J_{2} & 0 \\
0 & 0 & J_{3}
\end{array}\right]
$$

Three different coordinate reference frames are used. Let $N$ be the inertial reference frame, $B$ be the body reference frame attached to the principal inertia axes of the spacecraft, $R$ be the reference frame of the instantaneous reference motion that the spacecraft is tracking. Then the vector $\vec{\omega}_{B / N}$ describes the body angular velocity of the body frame relative to the inertial reference frame. Let the $\vec{\Omega}$ vector contain the angular velocities of the reaction wheels, measured relative to the $B$ frame. The rotational spacecraft equations of motion can be written as 2,3

$$
\Im \frac{d \vec{\omega}_{B / N}}{d t}=-\left[\tilde{\omega}_{B / N}\right] \Im \vec{\omega}_{B / N}-\left[\tilde{\omega}_{B / N}\right] J\left(\vec{\Omega}+\vec{\omega}_{B / N}\right)-\vec{u}+\vec{f}
$$

where the control vector $\vec{u}$ also satisfies the reaction axial wheel equation of motion:

$$
\vec{u}=J\left(\frac{d \vec{\Omega}}{d t}+\frac{d \vec{\omega}_{B / N}}{d t}\right)
$$

The tilde matrix $[\tilde{\omega}]$ is defined as

$$
[\tilde{\omega}]=\left[\begin{array}{ccc}
0 & -\omega_{3} & \omega_{2} \\
\omega_{3} & 0 & -\omega_{1} \\
-\omega_{2} & \omega_{1} & 0
\end{array}\right]
$$

and the vector $\vec{f}$ is the sum of all external torques acting on the spacecraft. These torques are in part due to aerodynamic and solar radiation drag and are usually considered to be very small and slowly time varying compared to the internal torques being applied.

\section{Attitude Coordinates}

All spacecraft orientations are described using sets of modified Rodrigues parameters ${ }^{4-9}$. They are a non-singular, minimal coordinate representation of rigid body attitudes with several useful attributes. They can be defined as a transformation from the Euler parameters as

$$
\sigma_{i}=\frac{\beta_{i}}{1+\beta_{0}} \quad i=1,2,3
$$


or in terms of the principal rotating axis $\hat{e}$ and the principal rotating angle $\phi$ as

$$
\vec{\sigma}=\hat{e} \cdot \tan \phi / 4
$$

Like the Euler parameters, the modified Rodrigues parameters (MRPs) are not unique. A second set of modified Rodrigues parameters, called the "shadow" set, can be used to avoid the singularity at the cost of a discontinuity at a switching point. The transformation between the "original" and "shadow" sets of MRPs is 4,6,7,9

$$
\sigma_{i}^{S}=-\sigma_{i} / \vec{\sigma}^{T} \vec{\sigma} \quad i=1,2,3
$$

Keep in mind that the choice in distinguishing "original" and "shadow" set is purely arbitrary. both sets describe the same physical orientation. In this study the switching condition was chosen to be $\vec{\sigma}^{T} \vec{\sigma}=1$. This causes the magnitude of the orientation vector to be bounded between $0 \leq \vec{\sigma} \leq 1$ and the principal rotation angle to be restricted between $-180^{\circ} \leq \phi \leq+180^{\circ}$. Note that this combined set of "original" and "shadow" parameters implicitly "knows" the shortest way back to the origin ${ }^{4}$. Principal rotations of more than $180^{\circ}$ are typically avoided. This is useful when designing a robust attitude feedback control law to detumble a spacecraft.

The differential kinematic equations of motion in terms of the modified Rodrigues parameters are given below $4,5,9$. The equation only contains second order polynomial nonlinearities in $\vec{\sigma}$.

$$
\frac{d \vec{\sigma}}{d t}=\frac{1}{2}\left[I\left(\frac{1-\vec{\sigma}^{T} \bar{\sigma}}{2}\right)+[\tilde{\sigma}]+\vec{\sigma} \bar{\sigma}^{T}\right] \vec{\omega}
$$

Eq. (8) holds for both the "original" and the "shadow" set. This means that the derivative is well defined even at the switching point. The direction cosine matrix in terms of the modified Rodrigues parameters is $4,5,9$

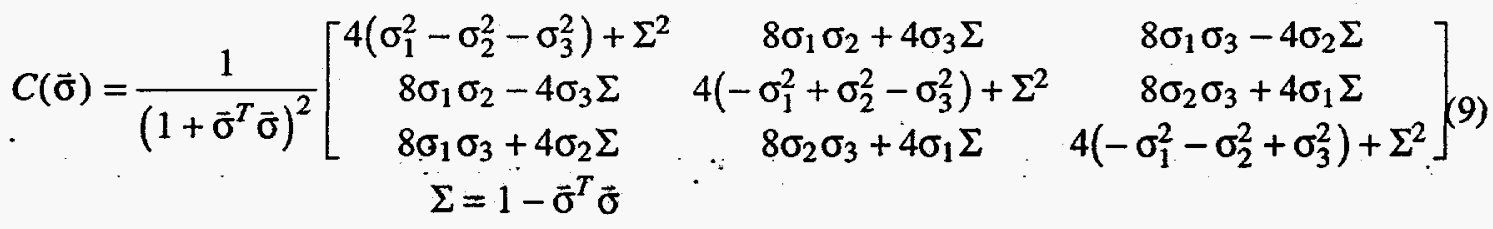

\section{ADAPTIVE EXTERNAL TORQUE ESTIMATOR}

This paper will use the globally asymptotically stable nonlinear tracking control law from the paper by Schaub, Robinett and Junkins ${ }^{1}$ to present and demonstrate the adaptive external torque estimation method. Please note that this method is not tied to this particular Lyapunov function, but it should work with most Lyapunov derived control laws.

The direction cosine matrix $[B R]$ defines the relative attitude of the body frame to the reference frame. Let the modified Rodrigues parameter vector $\vec{\sigma}$ parameterize the $[B R]$ matrix. This vector defines the spacecraft attitude error. Achieving $\vec{\sigma} \rightarrow 0$ assumes asymptotic tracking of the reference motion. The extraction of the $\bar{\sigma}$ vector from the $[B R]$ matrix is easily accomplished by use of the $\beta_{0}$ Euler parameter. The complete transformation is given Eq. (10) for the case where $\beta_{0}>0$. A general transformation which holds for the case where $\beta_{0}=0$ is to extract the principal rotation vector and angle from $[B R]$ and use Eq. (6) to find the modified Rodriguez vector. 


$$
\begin{aligned}
2 \beta_{0} & =+\sqrt{\operatorname{trace}([B R])+1} \\
\sigma_{1} & =\frac{B R_{23}-B R_{32}}{4 \beta_{0}\left(1+\beta_{0}\right)} \\
\sigma_{2} & =\frac{B R_{31}-B R_{13}}{4 \beta_{0}\left(1+\beta_{0}\right)} \\
\sigma_{3} & =\frac{B R_{12}-B R_{21}}{4 \beta_{0}\left(1+\beta_{0}\right)}
\end{aligned}
$$

By assuring that $\beta_{0} \geq 0$ we are guaranteed to have a modified Rodrigues parameter vector ${ }^{4}$ with $|\vec{\sigma}| \leq 1$. Let all the following vectors be written in the body frame $B$, unless noted by a superscript letter otherwise. The error in body angular velocity with components in body axes is

$$
\delta \vec{\omega}=\vec{\omega}_{B / N}-[B R] \vec{\omega}_{R / N}^{R}
$$

The error in body angular acceleration is found by taking the derivative of Eq. (11).

$$
\frac{d}{d t}(\delta \vec{\omega})^{N}=\frac{d}{d t}\left(\vec{\omega}_{B / N}\right)^{N}-[B R] \frac{d}{d t}\left(\vec{\omega}_{R / N}\right)^{N}+\left[\tilde{\omega}_{B / N}\right][B R] \vec{\omega}_{R / N}^{R}
$$

Reference 1 introduces the following Lyapunov function to study global stability of feedback controlled motion.

$$
V=\frac{1}{2} \delta \vec{\omega}^{T} \Im \delta \vec{\omega}+2 K \log \left(1+\vec{\sigma}^{T} \vec{\sigma}\right)
$$

The attitude feedback gain $K$ is a scalar. Using the logarithm of the departure motion will result in a feedback control law which is linear in $\vec{\sigma}^{1,4,5}$. The feedback/feedforward control law below is the same as in Ref. 1 except for the estimated external torque $\hat{f}$ term.

$$
\begin{aligned}
\vec{u}= & -\mathfrak{J}\left([B R] \frac{d}{d t}\left(\bar{\omega}_{R / N}^{R}\right)^{N}-\left[\tilde{\omega}_{B / N}\right][B R] \vec{\omega}_{R / N}^{R}\right) \\
& -\left[\tilde{\omega}_{B / N}\right] \Im \vec{\omega}_{B / N}-\left[\tilde{\omega}_{B / N}\right] J\left(\vec{\Omega}+\bar{\omega}_{B / N}\right)+K \bar{\sigma}+P \delta \bar{\omega}+\hat{f}
\end{aligned}
$$

The matrix $P$ is the angular velocity feedback gain matrix and is positive definite. Similar to Ref. 1, the first derivative of the Lyapunov function $V$ is now reduced to

$$
\dot{V}=-\delta \bar{\omega}^{T} P \delta \bar{\omega}-\delta \bar{\omega}^{T}(\hat{f}-\vec{f})
$$

Let the estimated external torque be related to the actual external torque through

$$
\hat{f}=\vec{f}+\Delta \vec{f}
$$

where $\Delta \vec{f}$ is the external torque estimation error. Eq. (15) can now be rewritten as

$$
\dot{V}=-\delta \bar{\omega}^{T} P \delta \vec{\omega}-\delta \bar{\omega}^{T} \Delta \vec{f}
$$

Note that in the absence of an external torque estimation error $\Delta \vec{f}$, this system is guaranteed globally asymptotically stable ${ }^{1}$. When bounded unmodeled torques are added, but not compensated for in the feedback control law, the system is no longer asymptotic stable. In this case Eq. (17) only states that $|\delta \vec{\omega}| \rightarrow \infty$ as $t \rightarrow \infty$. As $|\delta \vec{\omega}|$ grows, $\dot{V}$ is guaranteed to become negative. 
Substituting the control torque $\vec{u}$ into the spacecraft equations of motion and using Eq. (12), the following closed-loop equations for the tracking error are obtained. To simplify notation let the 'symbol denote $d / d t()^{N}$.

$$
\begin{gathered}
\Im \delta \vec{\omega}^{\prime}=-K \vec{\sigma}-P \delta \vec{\omega}-\Delta \vec{f} \\
\vec{\sigma}^{\prime}=\frac{1}{2}\left[I\left(\frac{1-\vec{\sigma}^{T} \vec{\sigma}}{2}\right)+[\tilde{\sigma}]+\vec{\sigma} \vec{\sigma}^{T}\right] \delta \vec{\omega}=g(\vec{\sigma}) \delta \vec{\omega}
\end{gathered}
$$

The function $g(\bar{\sigma})$ is the $3 \times 3$ matrix coefficient of $\delta \bar{\omega}$ in Eq. (19). The two first order differential equations can be combined into one second order differential equation by taking the derivative of Eq. (18) and substituting Eq. (19) into it.

$$
\Im \delta \bar{\omega}^{\prime \prime}+P \delta \bar{\omega}^{\prime}+K g(\vec{\sigma}) \delta \bar{\omega}=-\Delta \vec{f}^{\prime}=-\left(\hat{f}^{\prime}-\vec{f}^{\prime}\right)=0
$$

Here it is assumed that the external torque $\vec{f}$ is constant and that the estimated external torque $\hat{f}$ does not get updated. Except for the $g(\vec{\sigma})$ term, these equations looks like the standard unforced oscillator equation. Because $\bar{\sigma} \in L_{\infty}$ by definition we have $g(\bar{\sigma}) \in L_{\infty}$. Since Eq. (20) has a constant damping term $P$ and we know from Eq. (17) that $\delta \bar{\omega} \in L_{\infty}$, the body angular velocity error must settle on a finite constant steady-state value $\delta \bar{\omega}_{s s}$. Setting the derivatives in Eq. (20) to zero we obtain

$$
K g(\vec{\sigma}) \delta \vec{\omega}_{s s}=0
$$

Since the $g(\bar{\sigma})$ matrix is always invertible ${ }^{6}$, the steady-state body angular velocity error is zero. Therefore $\delta \vec{\omega} \rightarrow 0$ as $t \rightarrow \infty$. To find the attitude tracking error as $t \rightarrow \infty$, take the limit of Eq. (18).

$$
\lim _{t \rightarrow \infty}\left(\mathfrak{I} \delta \bar{\omega}^{\prime}\right)=\lim _{t \rightarrow \infty}(-K \vec{\sigma}-P \delta \bar{\omega}-\Delta \vec{f})
$$

Since we know that the body angular velocity error will decay to zero, and $\Delta \bar{f}$ is assumed to be constant, the attitude error will converge to the following finite offset.

$$
\lim _{i \rightarrow \infty} \vec{\sigma}=-\frac{\Delta \vec{f}}{K}
$$

Therefore the control law in Eq. (14) only makes the system Lagrange stable or bounded in the presence of bounded unmodeled external torques. In particular, the attitude error will not converge to zero but to a finite offset. This offset can be reduced to be within acceptable limits by increasing the attitude feedback gain $K$. However, increasing this gain will limit the error recovery performance of the feedback law by saturating the reaction wheels sooner.. Of course $\vec{f}$ need not be constant, only slowly varying compared to the estimator and controller time constants, in order that the above analysis has practical significance.

For high precision attitude tracking, this small attitude offset or the high gain $K$ may not be good enough. The external torque estimate needs to be updated in a robust manner for $\Delta \vec{f} \rightarrow 0$. The following adaptive external torque estimation method is based on the idea that system error dynamics should correspond exactly to the time history of the Lyapunov function $V$. Any deviation from this time history is assumed to be due to an error in the external torque estimate. While $\Delta \vec{f}$ is unknown, the Lyapunov function $V$ is known at any point in time since it only depends on 
the tracking errors. By comparing the theoretical Lyapunov function time history to measurement-based estimate of theLyapunov function, an update $\Delta \vec{f}$ is found that would force the true Lyapunov time history to track the theoretical counterpart. Eq. (17) could be used to solve for $\Delta \vec{f}$. But $\dot{V}$ cannot be calculated directly, only $V(\vec{\sigma}, \vec{\omega})$ can be estimated easily from the measurements of $\delta \vec{\omega}$ and $\vec{\sigma}$. Therefore $\dot{V}$ would have to be obtained, in essence, through numerical differentiation of $\mathrm{V}$. This is very undesirable, since numerical differentiation is unreliable when significant measurement noise is included. To write Eq. (17) in terms of $V$ and not $\dot{V}$, the equation is integrated over the time interval $\left[t_{1}, t_{2}\right]$.

$$
\int_{t_{1}}^{t_{2}}\left(\delta \vec{\omega}^{T} \Delta \vec{f}\right) d t=-\int_{t_{1}}^{t_{2}}\left(\delta \vec{\omega}^{T} P \delta \vec{\omega}+\dot{V}\right) d t
$$

Since the unmodeled external torque is assumed to be piece-wise constant over the time interval $\left[t_{1}, t_{2}\right]$, the estimation error $\Delta \vec{f}$ can also be assumed to be piece-wise constant. This assumption allows it to be moved outside the integration.

$$
\left(\int_{t_{1}}^{t_{2}} \delta \bar{\omega} d t\right)^{T} \Delta \vec{f}=-\int_{t_{1}}^{t_{2}} \delta \vec{\omega}^{T} P \delta \bar{\omega} d t-\int_{t_{1}}^{t_{2}} \dot{V} d t
$$

The right-most integration in Eq. (25) can be solved exactly. The result is an equation depending on the true Lyapunov function $V_{\text {true }}$ at different points in time, and not on $\dot{V}$.

$$
\left(\int_{t_{1}}^{t_{2}} \delta \vec{\omega} d t\right)^{T} \Delta \vec{f}=-\int_{t_{1}}^{t_{2}} \delta \vec{\omega}^{T} P \delta \vec{\omega} d t-\left(V_{\text {true }}\left(t_{2}\right)-V_{\text {true }}\left(t_{1}\right)\right)
$$

Let us rewrite the above in equation in the more compact notation below

$$
\vec{\eta}\left(t_{1}, t_{2}\right)^{T} \Delta \vec{f}=\gamma\left(t_{1}, t_{2}\right)
$$

where $\gamma \in \Re$ is a scalar quantity. Since $\Delta \vec{f}$ is a $3 \times 1$ vector, Eq. (27) cannot be solved uniquely. One possible solution is to write Eq. (27) for at least three distinct adjacent time intervals. Now the linear system of equations can be solved in a least-squares fashion. A major draw-back to this method, however, is that the least-squares inverse is not defined when the spacecraft performs a single axis rotation about one of its principal axes! Using a singular value decomposition routine to perform the inverse resolves the singularity problem, but the sets of equations are found to be extremely sensitive to angular velocity measurement noise. Even small noise rendered the adjustments totally unusable.

To avoid the above difficulty, a minimum-norm inverse method ${ }^{10}$ using only one time interval was used. The minimum $\|\Delta \vec{f}\|$ satisfying Eq. (27) is found to be

$$
\Delta \vec{f}_{\min }=\vec{\eta} \cdot \frac{\gamma}{\vec{\eta}^{T} \vec{\eta}}
$$

This $\Delta \vec{f}$. estimation will yield the most conservative (i.e. smallest in magnitude) correction satisfying Eq. (27). Contrary to the least-squares inverse method, it performs very well when the spacecraft performs a pure rotation about one of its principal axes. We find one minor problem, however, when $\vec{\eta}\left(t_{1}, t_{2}\right) \rightarrow 0$. This occurs when the body angular velocity error is very small on the average over the integration interval. We find that a simple and effective way to eliminate this 
difficulty is to not use the $\Delta \vec{f}$ correction when $\vec{\eta}$, on the average, is smaller than the RMS of the expected measurement noise of the body angular velocity. Let $\Delta \vec{f}_{\min }$ be defined as

$$
\Delta \vec{f}_{\text {min }}=\left\{\begin{array}{cc}
\vec{\eta} \cdot \frac{\gamma}{\vec{\eta}^{T} \vec{\eta}} & \text { if } \frac{|\vec{\eta}|}{t_{2}-t_{1}}>R M S\left(\omega_{\text {noise }}\right) \\
0 & \text { if } \frac{|\vec{\eta}|}{t_{2}-t_{1}} \leq R M S\left(\omega_{\text {noise }}\right)
\end{array}\right.
$$

If $\vec{\eta}$ is of the order of magnitude of the body angular velocity measurement noise, then the external torque estimate must already be very good. Turning the correction off does not affect performance in this case. This estimation dead-band has been found to be very effective in the examples studied.

The adaptive external torque estimation provides a real-time method to reduce the tracking errors in the presence of modeling errors. The computational burden to compute Eq. (26) and (29) is minimal. Unlike a Kalman filter, the method has little memory and is more easily able to adapt to a changing external torque. The adaptation is only affected by disturbances experienced during the integration interval.

If the actual external torque is constant, then $\Delta \vec{f} \rightarrow 0$ and therefore the attitude tracking error will also go to zero. For non-constant external torques, the length of the integration time interval in Eq. (26) will determine the tracking precision of the adaptive external torque estimation. Of course, the integration time must in reality be matched to sensor sample rates and signal processing speed, so there will be application specific practical limits on this approach.

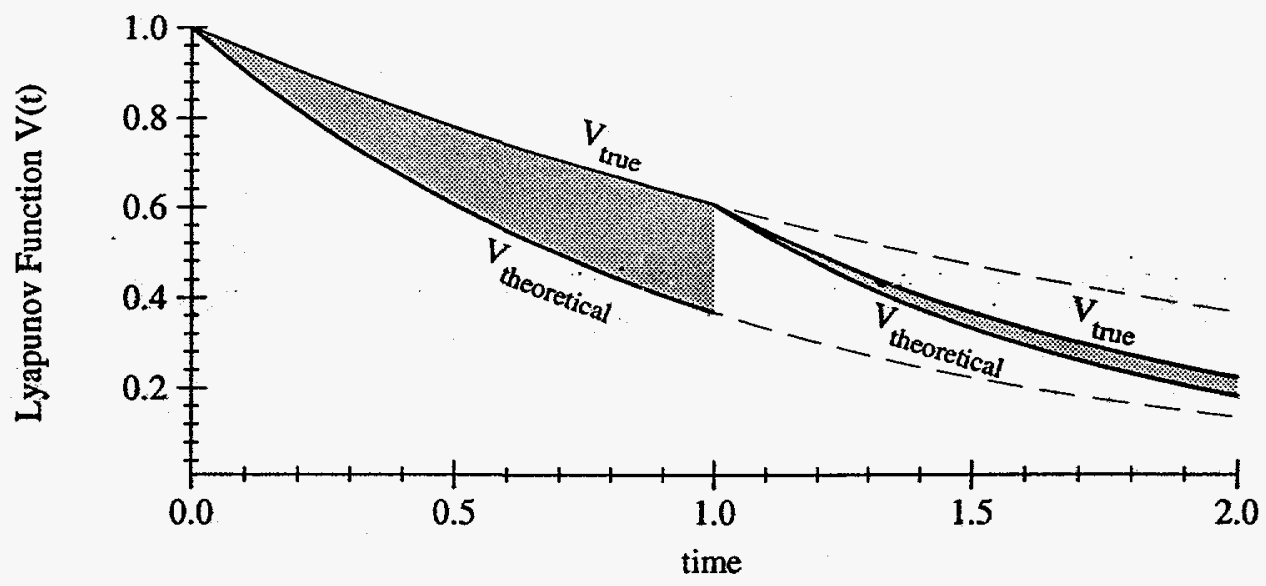

Figure 1: True versus Theoretical Lyapunov Function

The result of the update law in Eq. (29) is illustrated in Figure 1 above. Given a specific set of tracking errors, there are two Lyapunov function time histories. Vtrue corresponds to the actual error dynamics which may not decay to zero. Vtheoretical corresponds to the theoretical error dynamics of the current system model which is guaranteed to converge to zero. The shaded area represents the difference between the two time histories which is used to calculate the update of the model error. Since the system of equations in Eq. (27) is underdetermined, the update will not perfect. However, during the next integration integral the modeling error has been reduced. The $\Delta \vec{f}$ corrections force the tracking error dynamics to follow the time history of the Lyapunov function 
$V$ given in Eq. (13). Any deviation from this time history is interpreted as being caused by an unmodeled external torque and a correction is made to account for it. Other modeling errors which would cause the error dynamics to deviate from the Lyapunov function time history are absorbed into the $\Delta \vec{f}$ adjustments. By doing so, we find that this method is very robust versus spacecraft modeling errors and it improves the tracking performance greatly.

In a practical situation, the integrals of Eq. (26) cannot be computed exactly. The error in body angular velocity is only available at discrete sampling intervals. To account for this, let the integration interval $\left[t_{1}, t_{2}\right]$ span a discrete number of sampling intervals. A numerical integration technique, such as the trapezoidal rule, can then be used to approximate this integral. Naturally, the resulting integration error will degrade the estimation capability of the method. But the results below indicate that this integration error does not pose any significant limitations.

\section{ADAPTIVE LAW STABILITY ANALYSIS}

For this stability analysis it is assumed that the true external torque is constant. All possible $\Delta \vec{f}$ satisfying Eq. (27) form a linear variety $M^{10}$. Since the $\Delta \vec{f}$ vector lies in the Euclidean three-dimensional space, $M$ is simply a three-dimensional plane which generally does not go through the origin as illustrated in Figure 2 below.

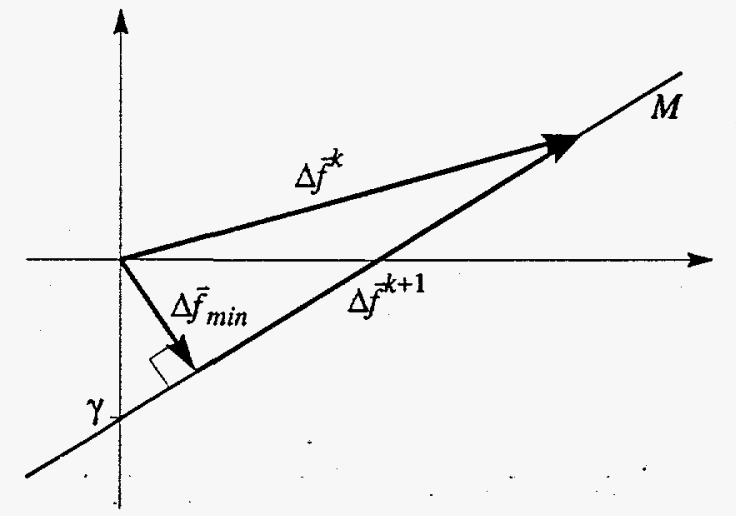

Figure 2: Illustration of the Minimum Norm Inverse

Let $\Delta \vec{f}^{k} \in M$ be the true external torque estimation error at the iteration $k$. Then the minimum norm inverse will return $\Delta \vec{f}_{\min } \in M$ which is perpendicular to the plane formed by $M^{10}$. After the update, the true external torque estimation error $\Delta \vec{f}^{k+1}$ is

$$
\Delta \bar{f}^{k+1}=\Delta \vec{f}^{k}-\Delta \bar{f}_{\min }
$$

Since $\Delta \vec{f}^{t+1}$ and $\Delta \vec{f}_{\min }$ are perpendicular to each other, the updated external torque estimate must satisfy

$$
\left\|\Delta \vec{f}^{k+1}\right\|<\left\|\Delta \vec{f}^{k}\right\| \text { for } \gamma \neq 0
$$

The above condition guarantees that the model error can only get reduced or remain the same, but cannot grow larger if the true external torque is constant. The only way that the model error would remain constant is that $\gamma=0$, where $\gamma$ is defined in Eq. (27). 


$$
\gamma=0 \Leftrightarrow \Delta \vec{f}^{k+1}=\Delta \vec{f}^{k}, \Delta \vec{f}_{\text {min }}=0
$$

Studying Eq. (27) it is clear that there are only three cases where $\gamma=0$.

Case 1: $\quad \Delta \vec{f}=0$

This is the ideal case where there is no model error. The update law has achieved its goal and estimated the external perfectly. No further update is required.

Case 2: $\quad \vec{\eta}=0$ and $\Delta \vec{f} \neq 0$

This condition would result if

i) $\int_{t_{2}}^{t_{2}} \delta \vec{\omega} d t=0$ and $\delta \vec{\omega}(t) \neq 0$. This is only possible if the vector components of $\delta \vec{\omega}$ change sign during the integration. The frequency of the error dynamics was set by choosing the $K$ and $P$ feedback gains. This frequency is usually set much slower than the sampling frequency. Therefore the $\delta \bar{\omega}$ components cannot change sign during every integration interval. Thus this case can only occur point wise in time. In between each occurrence the model error is guaranteed to get reduced by Eq. (31).

ii) $\delta \vec{\omega}(t)=0$. This means that the body angular velocity error is zero and remains zero from here on in time. If this is the case, then Eq. (26) dictates that $V_{\text {true }}\left(t_{2}\right)=V_{\text {true }}\left(t_{1}\right)$. The definition of the Lyapunov function in Eq. (13) states that this is only possible if $\vec{\sigma}(t)$ is a constant. Earlier it was shown that for constant external torque estimation error this is only possible if

$$
\vec{\sigma}=-\frac{\Delta \vec{f}}{K} \quad \text { and } \delta \vec{\omega}=0
$$

If the initial tracking errors are such that the conditions in Eq. (33) are satisfied, then the update law in Eq. (28) would not reduce the model error. However, for a real system where measurement noise and unmodeled dynamics are present, this condition cannot hold for all time. Further, these tracking errors defined in Eq. (33) are not stable either. As soon as the external torque estimate gets updated, the condition is no longer true. This means that the model errors becomes further reduced and $\delta \vec{\omega}(t)$ is no longer zero.

Case 3: $\quad \vec{\eta}^{T} \Delta \vec{f}=0$ and $\vec{\eta}, \Delta \vec{f} \neq 0$

This condition implies that the body angular velocity error is in a plane perpendicular to the external torque estimation error as shown in Figure 3 below.

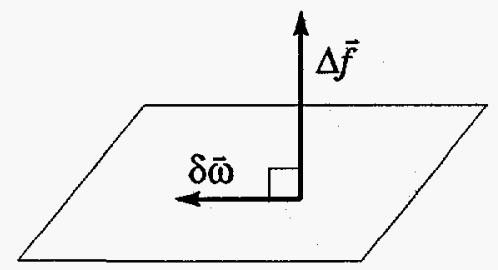

Figure 3: Illustration of the Condition in Case 3

Since there is an external torque error which is not modelled in the error dynamics, it is impossible for the $\delta \bar{\omega}$ vector to remain in this plane. As soon as it leaves the update law will change the $\Delta \vec{f}$ vector. Therefore, this condition is also only point-wise possible in time. 
Assuming constant external torque, the adaptive law in Eq. (28) is guaranteed to drive the external torque estimate error to zero, making the tracking error asymptotically stable, in all cases except case 2.ii). It is possible that the update law may temporarily cease to improve the estimation error, but it is not possible that cases 2.i) and 3) hold for all time. The condition in Case 2.ii) is not an attractor. For a real system with measurement noise and unmodeled dynamics the tracking error dynamics will move away from it and thus drive the external torque estimation error to zero.

\section{RESULTS}

A rest-to-rest near-minimum-time rotation ${ }^{1-3}$ is performed from the 3-2-1 Euler angles $\left(5^{\circ},-10^{\circ}, 15^{\circ}\right)$ to $\left(15^{\circ}, 10^{\circ},-5^{\circ}\right)$. The diagonal spacecraft inertia matrix entries used are $385 \mathrm{kgm}^{2}$, $398 \mathrm{kgm}^{2}$ and $212 \mathrm{kgm}^{2}$. The reaction wheel inertias are $0.06 \mathrm{kgm}^{2}, 0.12 \mathrm{kgm}^{2}$ and $0.06 \mathrm{kgm}^{2}$. A constant unmodeled external torque vector of $(0.01,0.02,0.01) \mathrm{Nm}$ is applied to the rigid spacecraft during the rotations. The attitude feedback gain $K$ is set to 5 and the body angular velocity feedback gain matrix $P$ has the diagonal entries $26.68,27.58$ and 14.69. The total maneuver time is 48.2 seconds. A fourth-order Runge-Kutta method was used in the simulation with a step size of 0.1 seconds. Equation (26) was integrated using the trapezoidal rule over two sampling intervals. The angular velocity measurement noise has a standard mean of $0.00004 \%$. To keep the illustrations simple only the magnitude of the tracking error vectors are plotted below.

\section{No External Torque Estimation}

For comparison purposes, the first simulation was run with no external torque estimation and no body angular velocity measurement noise. The results are shown in Figure 4 below. The attitude error grows through out the maneuver. Eventually, the feedback stabilizes the attitude error at an offset of $|\vec{\sigma}|=0.00490$ or $1.123^{\circ}$. Clearly this large attitude tracking error would be unacceptable when precise tracking is required. The body angular velocity error also grows quite large, nearly reaching magnitudes of $0.035 \%$.
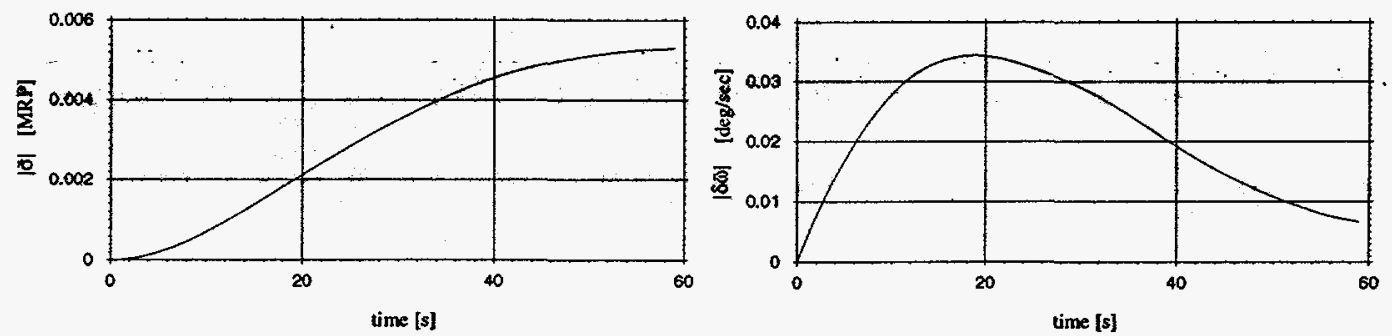

Figure 4: Tracking Errors with No External Torque Estimation and No Measurement Noise

\section{With External Torque Estimation}

The same simulation is repeated here with the adaptive external torque estimation activated. The integral in Eq. (26) is calculated over two sampling periods. The external torque estimate was set to zero initially. The results are shown in Figure 5 below. 

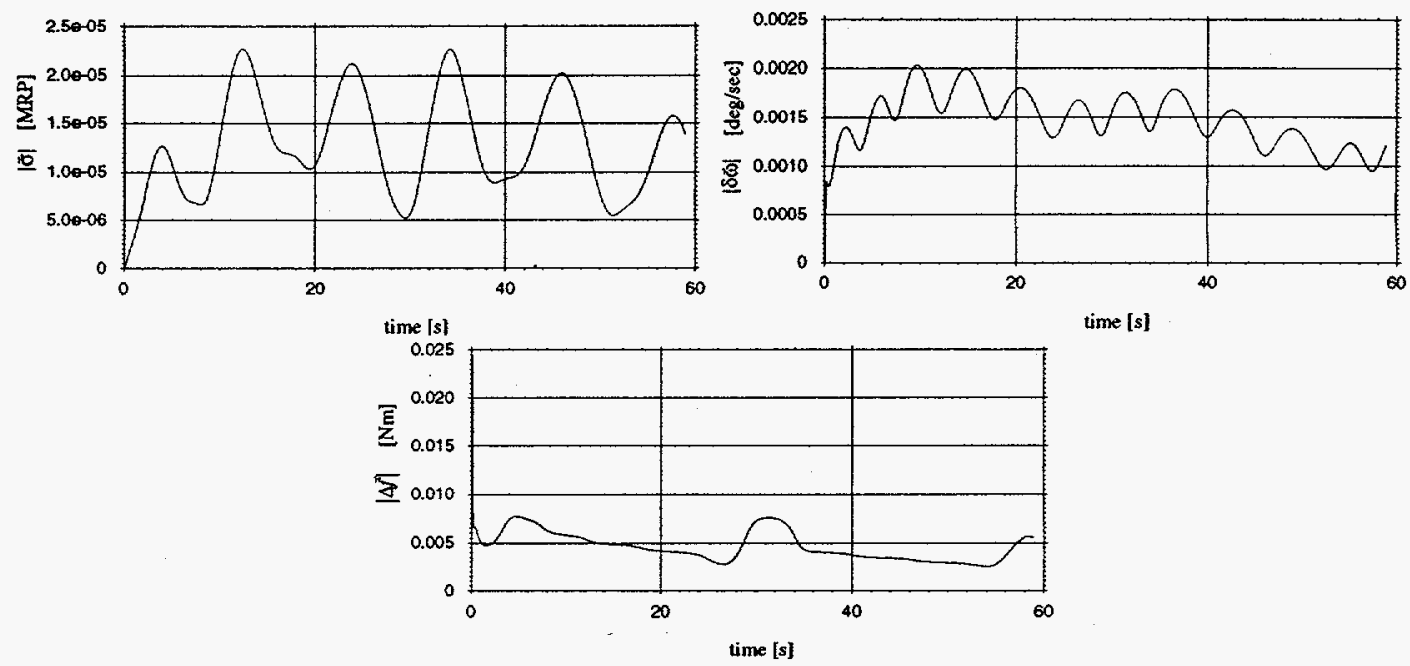

Figure 5: Tracking Errors with External Torque Estimation and No Measurement Noise

The attitude tracking error is reduced very significantly compared to the previous simulation with no disturbance estimation. The final tracking error is only $|\vec{\sigma}|=0.000015$ or $0.0034^{\circ}$. This is a more than two orders of magnitude reduction. The body angular velocity tracking error is also substantially reduced. On the average, the error is reduced by more than one order of magnitude. Both the attitude and the body angular velocity tracking errors oscillate about zero, not some finite offset. The oscillation about zero is not evident in Figure 2 since only the magnitudes of the tracking error vectors are shown. The error in external torque estimation drops very rapidly initially from a magnitude of $0.0245 \mathrm{Nm}$ to about $0.005 \mathrm{Nm}$ and then decays more slowly. This was to be expected since Eq. (27) is twice under determined. The "humps" in the decay curve are correlated to regions of sharp control input variations where both integration errors and system non-linearity are more pronounced. The actual decay rate could be increased with a higher body angular velocity sampling rate. Note that the external torque estimation corrections are very smooth. This makes this method attractive if the spacecraft has some unmodeled elastic degrees of freedom which are not to be excited by control input.

\section{Robustness in the Presence of Measurement Noise}

To evaluate the robustness of the adaptive external torque estimation method, a simulation was run with added body angular velocity measurement noise. The results are shown in Figure 6. Naturally, the body angular velocity error time history is affected by the measurement noise. Yet the external torque estimation error is only marginally affected by the noise. This is because most of the noise is partially filtered during the integration of Eq. (26). Had Eq. (17) been used to solve for $\Delta \vec{f}$, then the external torque estimation would have been very noisy due to the implicit requirement to estimate time derivatives of noisily measured quantities. 

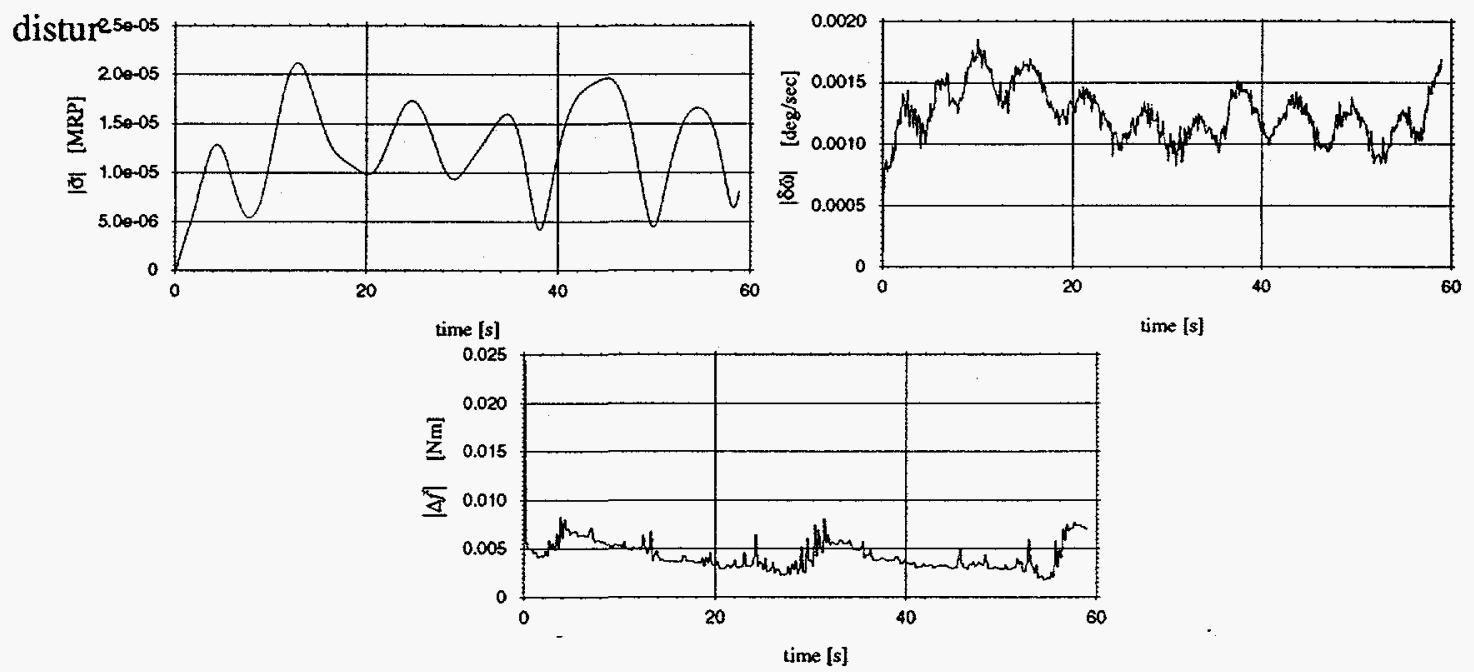

Figure 6: Tracking Errors with External Torque Estimation and Measurement Noise

\section{Robustness in the Presence of SpaceCraft Modeling Errors}

To study the effect of other modeling errors, the inertia matrix was given an error of $10 \%$. The results are shown in Figure 7. Without the adaptive external torque estimation, the attitude tracking error would be $|\vec{\sigma}|=0.006$ or $1.365^{\circ}$. With the external torque estimation active, as shown in Figure 7, the error is almost exactly two order of magnitude smaller. Similarly, the body angular velocity tracking error is reduced by about one order of magnitude.
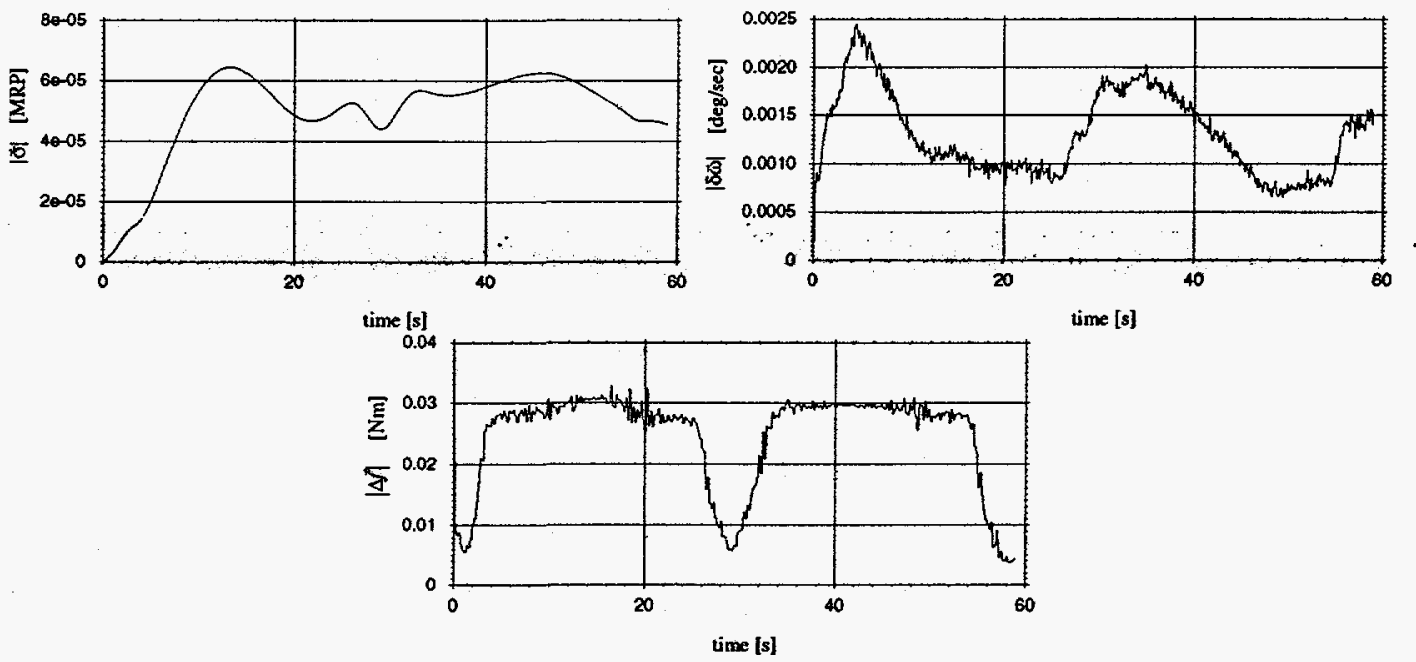

Figure 7: Tracking Errors with External Torque Estimation, Measurement Noise and 10\% Inertia Error

As one might anticipate, the effect of the incorrect inertias is absorbed and compensated for by the adaptive external torque estimation. This ability to compensate both for external 
and system model errors clearly makes this method very attractive, even when external torques are not an issue. In all cases of modeling error studied, the tracking error is greatly reduced.

With the large inertia errors present the external torque estimate error no longer approaches zero. Instead the inertia modeling error compensation dominates and successfully forces the tracking error dynamics to follow the Lyapunov function.

\section{Robustness in the Presence of a Time Varying External Torque}

To study how well the adaptive law can handle a time varying external torque, the same test run was performed as shown in Figure 6. This time the true external torque vector components were not constant but varied with a frequency of $0.04 \mathrm{~Hz}, 0.03 \mathrm{~Hz}$ and $0.05 \mathrm{~Hz}$ respectively. The resulting tracking errors are shown in Figure 8 below.
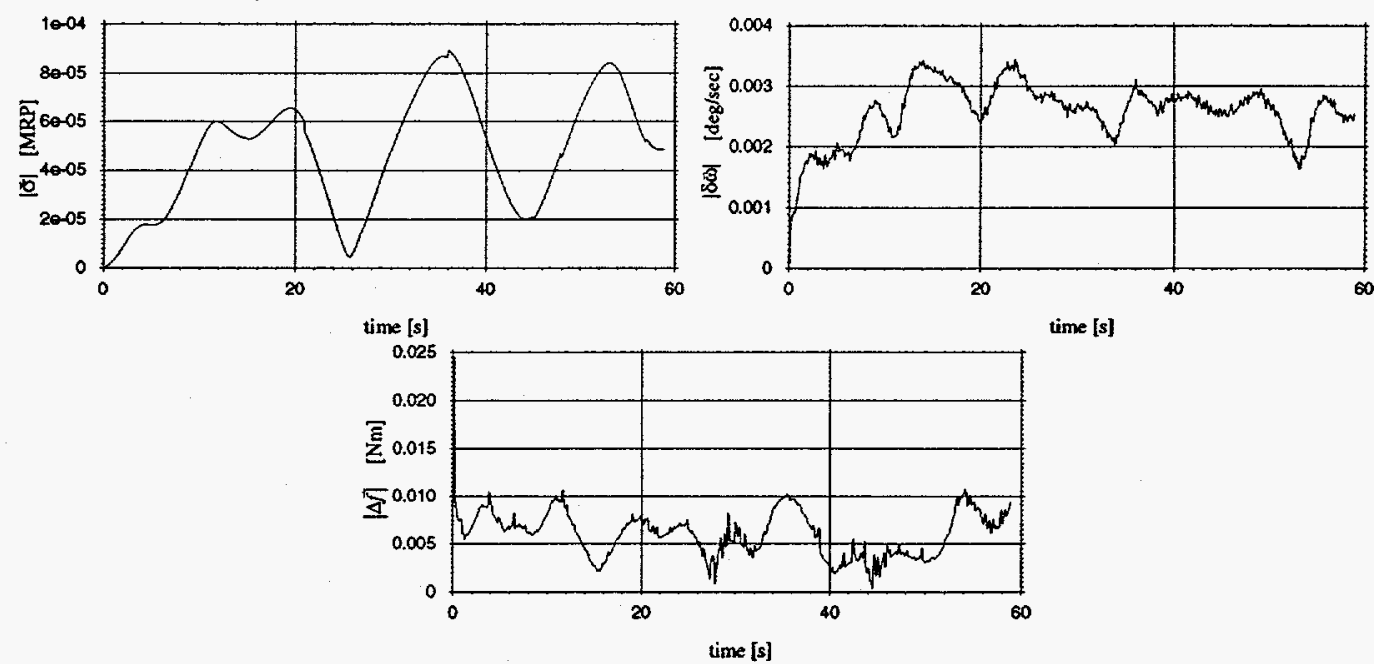

Figure 8: Tracking Errors with Measurement Noise and Time varying External Torque

The overall tracking errors are only marginally larger than those of Figure 6. The attitude and body angular velocity errors are still substantially reduced. The external torque estimation error no longer converges to zero, but remains bounded within a certain band.

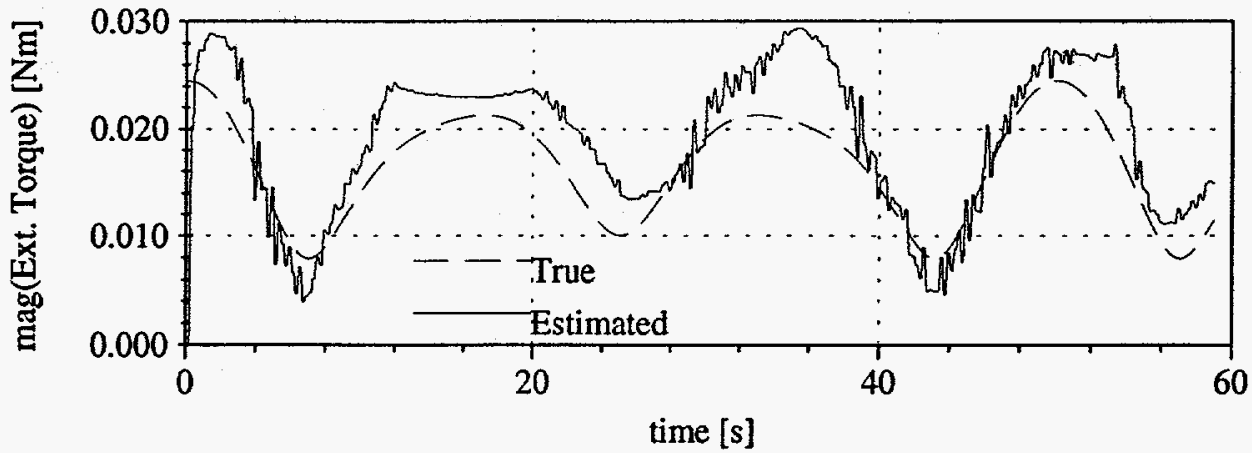

bances

Figure 9: Comparison of True and Estimated External Torque 
A comparison of the true and the estimated external torque magnitude can be seen in Figure 9. The adaptive law does a reasonable job tracking this very time varying external torque. Of course the tracking performance can always be improved by increasing the sampling frequency. If the sampling frequency is very low compared to the frequency of the external torque, the system may even go unstable.

\section{CONCLUSION}

To counter the effects of unmodeled external torques, an adaptive external torque estimator has been developed to operate in conjunction with a nominal asymptotically stable controller. The estimated external torque is adaptively adjusted to make the tracking error dynamics correspond to the Lyapunov function associated with the Lyapunov-designed control law. A minimum norm inverse is used to estimate external torque estimation errors. This adaptive technique works very well for all types of three-dimensional rotations studied. Since the body angular velocity errors get integrated (i.e. filtered) over finite time intervals, this method is also very robust versus measurement noise. For the test cases studied, the attitude tracking performance is increased by more then two orders of magnitude, while the body angular velocity tracking is improved by more than one order of magnitude. The method is also shown to be very robust in the presence of spacecraft modelling errors such as inertia errors. The external torque estimation actually is able to compensate for these modeling errors and can improve the tracking performance by roughly the same order of magnitudes as for the external torque compensation.

\section{REFERENCES}

[1] Schaub, H., Robinett, R. D., and Junkins, J. L., "Globally Stable Feedback Laws for Near-Minimum-Fuel and Near-Minimum-Time Pointing Maneuvers for a Landmark-Tracking Spacecraft," AAS/AIAA Astrodynamics Specialist Conference, Halifax, Nova Scotia, Canada, Aug. 14-17, 1995 paper 95-417.

[2] Junkins, J. L., and Kim, Y., Introduction to Dynamics and Control of Flexible Structures, AlAA Education Series; Washington D.C., 1993.

[3] Junkins, J .L, and Turner, J. D., Optimal Spacecraft Rotational Maneuvers, Elsevier Science Publishers, Netherlands, 1986.

[4] Schaub, H., and Junkins, J. L., "Stereographic Orientation Parameters for Attitude Dynamics: A Generalization of the Rodrigues Parameters," AAS/AIAA Spaceflight Mechanics Meeting, Albuquerque, New Mexico, Feb. 13-16, 1995, paper AAS 95-137.

[5] Tsiotras, P. "New Control Laws for the Attitude Stabilization of Rigid Bodies," Proceedings, IFAC Symposium on Automatic Control in Aerospace, Palo Alto, CA, Sept. 12-16, 1994, pp. 316-321.

[6] Schaub, H., Tsiotras, P., and Junkins, J. L., "Principal Rotation Representations of Proper NxN Orthogonal Matrices," to appear in International Journal of Engineering Science, 1994.

[7] Marandi, S. R., and Modi, V. J., "A Preferred Coordinate System and the Associated Orientation Representation in Attitude Dynamics," Acta Astronautica, Vol. 15, 1987, pp. 833-843.

[8] Wiener, T. F., "Theoretical Analysis of Gimballess Inertial Reference Equipment Using Delta-Modulated Instruments," Diss. Massachusetts Institute of Technology, March 1962. 
[9] Shuster, M. D., "A Survey of Attitude Representations," Journal of the Astronautical Sciences, Vol. 41, No. 4, 1993, pp. 439-517.

[10] Luenberger, D. G., Optimization by Vector Space Methods, John Wiley \& Sons, Inc., New York, 1969.

\section{DISCLAIMER}

This report was prepared as an account of work sponsored by an agency of the United States Government. Neither the United States Gnvernment nor any agency thereof, nor any of their employees, makes any warranty, express or implied, or assumes any legal liability or responsibility for the accuracy, completeness, or usefulness of any information, apparatus, product, or process disclosed, or represents that its use would not infringe privately owned rights. Reference herein to any specific commercial product; process, or service by trade name, trademark, manufacturer, or otherwise does not necessarily constitute or imply its endorsement, recommendation, or favoring by the United States Government or any agency thereof. The views and opinions of authors expressed herein do not necessarily state or reflect those of the United States Government or any agency thereof. 